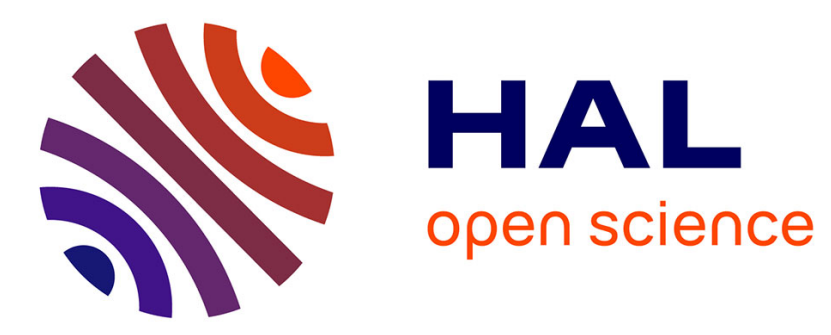

\title{
Quadratic spline quasi-interpolants and collocation methods
}

Françoise Foucher, Paul Sablonnière

\section{To cite this version:}

Françoise Foucher, Paul Sablonnière. Quadratic spline quasi-interpolants and collocation methods. Mathematics and Computers in Simulation, 2009, 79 (12), pp.3455-3465. 10.1016/j.matcom.2009.04.004 . hal-00468155

\section{HAL Id: hal-00468155 https://hal.science/hal-00468155}

Submitted on 30 Mar 2010

HAL is a multi-disciplinary open access archive for the deposit and dissemination of scientific research documents, whether they are published or not. The documents may come from teaching and research institutions in France or abroad, or from public or private research centers.
L'archive ouverte pluridisciplinaire HAL, est destinée au dépôt et à la diffusion de documents scientifiques de niveau recherche, publiés ou non, émanant des établissements d'enseignement et de recherche français ou étrangers, des laboratoires publics ou privés. 


\title{
Quadratic spline quasi-interpolants and collocation methods
}

\author{
Françoise Foucher ${ }^{a, *}$ and Paul Sablonnière ${ }^{\mathrm{b}}$ \\ ${ }^{a}$ Laboratoire de Mathématiques Jean Leray, Ecole Centrale de Nantes, BP 92101 \\ 44321 Nantes cedex 3, France \\ ${ }^{\mathrm{b}}$ Centre de mathématiques, INSA de Rennes, CS 14315, 35043 Rennes cedex, \\ France
}

\begin{abstract}
Univariate and multivariate quadratic spline quasi-interpolants provide interesting approximation formulas for derivatives of approximated functions that can be very accurate at some points thanks to the superconvergence properties of these operators. Moreover, they also give rise to good global approximations of derivatives on the whole domain of definition. From these results, some collocation methods are deduced for the solution of ordinary or partial differential equations with boundary conditions. Their convergence properties are illustrated and compared with finite difference methods on some numerical examples of elliptic boundary value problems.
\end{abstract}

Key words: spline approximants, Numerical differentiation, spline collocation methods.

1991 MSC: 65D07, 65D25, 65N35

\section{Introduction}

Univariate and multivariate spline quasi-interpolants (abbr. QIs) have been studied for several decades in approximation theory (see e.g. [1a, 1c, 2, 8, 9a, 9b, 9c]). However, few studies have been devoted to their potential applications in numerical analysis. Such a program has already been initiated in $[7 a-b]$ where some applications to the approximation of derivatives have been developed. In this paper, we focus on approximations of first and second

\footnotetext{
* Corresponding author. Tel.:(33) 2403725 19; fax : (33) 240372578

Email addresses: Francoise.Foucher@ec-nantes.fr,

Paul.Sablonniere@insa-rennes.fr (Paul Sablonnière).
} 
derivatives by those of quadratic spline quasi-interpolants and their applications to collocation methods. Though it is possible to use spline QIs of higher degrees and smoothness, we want to show that already simple $C^{1}$ quadratic splines provide rather good numerical methods.

Let $I=[a, b]$ with the uniform partition $\mathcal{X}_{n}=\left\{x_{i}=a+i h, 0 \leq i \leq n\right\}$ where $h=(b-a) / n$, and $x_{-2}=a-2 h, x_{-1}=a-h, x_{n+1}=b+h, x_{n+2}=b+2 h$. For $1 \leq i \leq n$, let $t_{i}=\frac{1}{2}\left(x_{i-1}+x_{i}\right), u_{i}=t_{i}-h r / 6, v_{i}=t_{i}+h r / 6$ (where $r:=\sqrt{3})$, and let $t_{0}=a, t_{n+1}=b, v_{0}=a, u_{n+1}=b$. Let $J:=\{0,1, \ldots, n+1\}$. On $\mathcal{T}_{n}:=\left\{t_{i}, i \in J\right\}$ we define $f_{i}=f\left(t_{i}\right), 0 \leq i \leq n+1$, and on the set of Gauss abscissas $\mathcal{G}_{n}:=\left\{v_{0} ; u_{i}, v_{i}, 1 \leq i \leq n ; u_{n+1}\right\}$, we define $\tilde{f}_{i}=f\left(u_{i}\right)$, $1 \leq i \leq n+1$ and $\hat{f}_{i}=f\left(v_{i}\right), 0 \leq i \leq n$. Quadratic B-splines $\left\{B_{i}, i \in J\right\}$, with supports $\left[x_{i-2}, x_{i+1}\right]$ form a basis of the $n+2$-dimensional space $\mathcal{S}_{2}\left(I, \mathcal{X}_{n}\right)$ of $C^{1}$ quadratic splines (see e.g. [1b, 3, 10]).

In Section 2, we study the uniform quadratic spline quasi-interpolant (abbr. uniform QI) $\bar{Q}$, and the Gauss quadratic spline quasi-interpolant (abbr. Gauss QI) $\widetilde{Q}$, respectively defined by

$$
\bar{Q} f=\sum_{i \in J} \lambda_{i}(f) B_{i} \quad \text { and } \quad \widetilde{Q} f=\sum_{i \in J} \mu_{i}(f) B_{i}
$$

where the coefficient functionals are given, for $2 \leq i \leq n-1$, by

$$
\lambda_{i}(f)=\frac{1}{8}\left(-f_{i-1}+10 f_{i}-f_{i+1}\right) \quad \text { and } \quad \mu_{i}(f)=\alpha\left(\tilde{f}_{i}+\hat{f}_{i}\right)+\beta\left(\hat{f}_{i-1}+\tilde{f}_{i+1}\right),
$$

with $\alpha=(9+r) / 12$ and $\beta=-(3+r) / 12$. For extreme indices, the functionals have specific forms (see Section 2 below). The uniform QI is based on the set $\mathcal{X}_{n}$ and the Gauss QI is based on the set $\mathcal{G}_{n}$. The choice of coefficients implies that the two QIs are exact on $\mathbb{P}_{2}$, i.e. $\bar{Q} m_{s}=\widetilde{Q} m_{s}=m_{s}$ for $s=0,1,2$, with the notation $m_{s}(x):=x^{s}$. This can be verified by using the B-spline representation of monomials. It implies in particular ([5], chapter 5), that the global approximation error $Q f-f$ on a smooth function $f$ is $O\left(h^{3}\right)$.

However, we notice that $Q f(a)=f(a), Q f(b)=f(b), Q f\left(x_{i}\right)-f\left(x_{i}\right)=O\left(h^{4}\right)$, $1 \leq i \leq n-1$, and $Q f\left(t_{i}\right)-f\left(t_{i}\right)=O\left(h^{4}\right), 1 \leq i \leq n$, for both quasiinterpolants $Q=\bar{Q}$ or $\widetilde{Q}$. Therefore there is a superconvergence phenomenon of these operators on the sets of points $\mathcal{X}_{n}$ and $\mathcal{T}_{n}$. This phenomenon does not hold on $\mathcal{G}_{n}$. On the other hand, in Section 3, we show that another superconvergence phenomenon takes place at Gaussian points for first derivatives, which leads to an improvement of global approximation properties of these derivatives. Section 4 describes some derivation matrices which are used in Section 5 in collocation methods for the solution of some univariate Dirichlet problems. The numerical results show an improvement with respect to classical finite difference methods. Finally, Sections 6 and 7 give some bivariate extensions of previous methods and an application to the Dirichlet problem for the Laplace equation. Once again, the new method is better than finite difference methods for the Laplacian. 


\section{Univariate quadratic spline quasi-interpolants}

\subsection{Properties of the uniform quasi-interpolant}

The specific coefficient functionals for extreme indices of the uniform quadratic spline quasi-interpolant $\bar{Q} f=\sum_{i \in J} \lambda_{i}(f) B_{i}$ are defined as follows :

$$
\begin{array}{ll}
\lambda_{0}(f):=\frac{12}{5} f_{0}-\frac{13}{8} f_{1}+\frac{1}{4} f_{2}-\frac{1}{40} f_{3}, & \lambda_{n+1}(f):=\frac{12}{5} f_{n+1}-\frac{13}{8} f_{n}+\frac{1}{4} f_{n-1}-\frac{1}{40} f_{n-2}, \\
\lambda_{1}(f):=-\frac{2}{5} f_{0}+\frac{13}{8} f_{1}-\frac{1}{4} f_{2}+\frac{1}{40} f_{3}, & \lambda_{n}(f):=-\frac{2}{5} f_{n+1}+\frac{13}{8} f_{n}-\frac{1}{4} f_{n-1}+\frac{1}{40} f_{n-2} .
\end{array}
$$

We notice that $\bar{Q} f(a)=\left(\lambda_{0}+\lambda_{1}\right) / 2=f_{0}=f(a)$ and $\bar{Q} f(b)=\left(\lambda_{n}+\lambda_{n+1}\right) / 2=$ $f_{n+1}=f(b)$, therefore $\bar{Q} f$ interpolates $f$ at the extreme points of $I$. This QI can also be written in the quasi-Lagrange form

$$
\bar{Q} f=\sum_{j \in J} f_{j} \bar{B}_{j}, \text { with } \bar{B}_{j}:=\frac{1}{8}\left(-B_{j-1}+10 B_{j}-B_{j+1}\right), 4 \leq j \leq n-3 \text {. }
$$

The coefficients in the basis $\left\{B_{i}, i \in J\right\}$ of the first and last four quasi-Lagrange functions are given in the following table (with upper (resp. lower) indices for left (resp. right) functions).

\begin{tabular}{|c|c|c|c|c|c|c|}
\hline$i$ & 0 & 1 & 2 & 3 & 4 & \\
\hline $\bar{B}_{0}$ & $12 / 5$ & $-2 / 5$ & 0 & 0 & 0 & $\bar{B}_{n+1}$ \\
\hline $\bar{B}_{1}$ & $-13 / 8$ & $13 / 8$ & $-1 / 8$ & 0 & 0 & $\bar{B}_{n}$ \\
\hline $\bar{B}_{2}$ & $1 / 4$ & $-1 / 4$ & $5 / 4$ & $-1 / 8$ & 0 & $\bar{B}_{n-1}$ \\
\hline $\bar{B}_{3}$ & $-1 / 40$ & $1 / 40$ & $-1 / 8$ & $5 / 4$ & $-1 / 8$ & $\bar{B}_{n-2}$ \\
\hline & $n+1$ & $n$ & $n-1$ & $n-2$ & $n-3$ & $i$ \\
\hline
\end{tabular}

Proposition 1. The quasi-interpolant $\bar{Q}$ is exact on the space $\mathbb{P}_{2}$ of quadratic polynomials, i.e. $\bar{Q} m_{r}=m_{r}$ for $r=0,1,2$. Moreover $\|\bar{Q}\|_{\infty}=3 / 2$.

Proof: Let us give the proof for $\bar{Q} m_{2}=m_{2}$. It is well known (see e.g. [1b]) that $m_{2}=\sum_{j \in J} x_{j-1} x_{j} B_{j}$. Using $t_{j-1}=t_{j}-h$ and $t_{j+1}=t_{j}+h$, we obtain

$$
\lambda_{j}\left(m_{2}\right)=\frac{1}{8}\left(-t_{j-1}^{2}+10 t_{j}^{2}-t_{j+1}^{2}\right)=t_{j}^{2}-\frac{h^{2}}{4}=x_{j-1} x_{j}, \text { for } 2 \leq j \leq n-1 .
$$


In the same way, one verifies that

$$
\begin{aligned}
& \lambda_{0}\left(m_{2}\right)=\frac{1}{40}\left(96 a^{2}-65\left(a+\frac{h}{2}\right)^{2}+10\left(a+\frac{3 h}{2}\right)^{2}-\left(a+\frac{5 h}{2}\right)^{2}\right)=x_{-1} x_{0}, \\
& \lambda_{1}\left(m_{2}\right)=2 m_{2}(a)-\lambda_{0}\left(m_{2}\right)=2 a^{2}-a(a-h)=x_{0} x_{1}, \\
& \lambda_{n}\left(m_{2}\right)=x_{n-1} x_{n} \quad \text { and } \quad \lambda_{n+1}\left(m_{2}\right)=x_{n} x_{n+1} .
\end{aligned}
$$

$\|\bar{Q}\|_{\infty}$ is equal to the Chebyshev norm of its quasi-Lagrange function $\bar{\Lambda}:=$ $\sum_{j \in J}\left|\bar{B}_{j}\right|$ and a direct study of the latter leads to $\|\bar{Q}\|_{\infty}=|\bar{\Lambda}|_{\infty}=\bar{\Lambda}\left(x_{1}\right)=$ $\bar{\Lambda}\left(x_{n-1}\right)=3 / 2$

Proposition 2. For all indices $i$, there holds $\bar{Q} f\left(x_{i}\right)-f\left(x_{i}\right)=O\left(h^{4}\right)$ and $\bar{Q} f\left(t_{i}\right)-f\left(t_{i}\right)=O\left(h^{4}\right)$, therefore $\bar{Q} f$ is superconvergent on both sets of points $\mathcal{X}_{n}$ and $\mathcal{T}_{n}$.

Proof: the superconvergence of $\bar{Q} f$ on $\mathcal{X}_{n}$ and $\mathcal{T}_{n}$ is verified by computing Taylor expansions on both sets of points with the help of a computer algebra system. Setting $\bar{E} f=\bar{Q} f-f$, we thus obtain successively

$$
\begin{aligned}
& \bar{E} f\left(t_{1}\right)=\bar{E} f\left(t_{n}\right)=0, \\
& \bar{E} f\left(t_{i}\right) \sim \bar{C}_{i} h^{4} D^{4} f\left(t_{i}\right) \text { where } \bar{C}_{2}=\bar{C}_{n-1}=\frac{7}{512}, \bar{C}_{i}=\frac{1}{64}, 3 \leq i \leq n-2, \\
& \bar{E} f\left(x_{i}\right) \sim \bar{C}_{i}^{\prime} h^{4} D^{4} f\left(x_{1}\right) \text { where } \bar{C}_{1}^{\prime}=\bar{C}_{n-1}^{\prime}=\frac{1}{64}, \bar{C}_{i}^{\prime}=\frac{3}{128}, 2 \leq i \leq n-2
\end{aligned}
$$

Remark 1. The quasi-interpolant $\bar{Q}$ studied here is slightly different from the quadratic spline QI already studied in [9b]. The first and last coefficient functionals $\lambda_{0}(f)$ and $\lambda_{n+1}(f)$ have been modified in order to get the superconvergence at points $t_{1}, x_{1}, t_{2}, t_{n-1}, x_{n}, t_{n}$. The norm is also a little bit bigger.

Finally, we consider the global approximation error $\bar{Q} f-f$, which is known to be $O\left(h^{3}\right)$ for functions $f \in C^{3}(I)$. In each subinterval $I_{i}:=\left[x_{i-1}, x_{i}\right]$, the local Lagrange interpolant $p_{i} \in \mathbb{P}_{2}$ on the set $T_{i}:=\left\{x_{i-1}, t_{i}, x_{i}\right\}$ satisfies for all $x \in I_{i}, f(x)-p_{i}(x)=\frac{1}{6}\left(x-x_{i-1}\right)\left(x-t_{i}\right)\left(x-x_{i}\right) D^{3} f(\xi)$, with $\xi \in I_{i}$. Therefore, an elementary calculation leads to $\max _{x \in I_{i}}\left|f(x)-p_{i}(x)\right| \leq \frac{r}{216} h^{3}\left\|D^{3} f\right\|_{\infty}$. On the other hand, setting $\bar{p}_{i}=\bar{Q} f_{\mid I_{i}}$, and denoting $\lambda_{i}$ for $\lambda_{i}(f)$, we obtain

$$
\bar{p}_{i}\left(x_{i-1}\right)=\frac{\lambda_{i-1}+\lambda_{i}}{2}, \bar{p}_{i}\left(t_{i}\right)=\frac{\lambda_{i-1}+6 \lambda_{i}+\lambda_{i+1}}{8}, \bar{p}_{i}\left(x_{i}\right)=\frac{\lambda_{i}+\lambda_{i+1}}{2} .
$$

Using Taylor expansions at $x_{i}$ with integral remainders, we get for example $\bar{p}_{i}\left(x_{i}\right)=\frac{1}{16}\left(-f\left(t_{i-1}\right)+9 f\left(t_{i}\right)+9 f\left(t_{i+1}\right)-f\left(t_{i+2}\right)\right)=f\left(x_{i}\right)+\int_{x_{i-1}}^{x_{i}} k_{i}(t) D^{3} f(t) d t$, where the Peano kernel $k_{i}(t)$ is a piecewise quadratic function on the four intervals $\left[t_{i-1}, t_{i}\right],\left[t_{i}, x_{i}\right],\left[x_{i}, t_{i+1}\right],\left[t_{i+1}, t_{i+2}\right]$. From that representation (and a 
similar one at $x_{i-1}$ ), and setting $\bar{e}_{i}:=f-\bar{p}_{i}$, we deduce the error bounds

$$
\left|\bar{e}_{i}\left(x_{i-1}\right)\right|,\left|\bar{e}_{i}\left(x_{i}\right)\right| \leq \frac{3 h^{3}}{64}\left\|D^{3} f\right\|_{\infty}, \quad\left|\bar{e}_{i}\left(t_{i}\right)\right| \leq \frac{h^{3}}{48}\left\|D^{3} f\right\|_{\infty} .
$$

The Lagrange basis $\left\{\ell_{0}, \ell_{1}, \ell_{2}\right\}$ of $\mathbb{P}_{2}$ based on $T_{i}$ satisfying $\left\|\sum_{k=0}^{2}\left|\ell_{k}\right|\right\|_{\infty}=\frac{5}{4}$, we then obtain

$$
\left|\left(p_{i}-\bar{p}_{i}\right)(x)\right|=\left|\bar{e}_{i}\left(x_{i-1}\right) \ell_{0}(x)+\bar{e}_{i}\left(t_{i}\right) \ell_{1}(x)+\bar{e}_{i}\left(x_{i}\right) \ell_{2}(x)\right| \leq \frac{15}{256} h^{3}\left\|D^{3} f\right\|_{\infty} .
$$

Finally we deduce from the two above majorations

Proposition 3. When $D^{3} f$ is bounded, the following error estimate holds:

$$
\|f-\bar{Q} f\|_{\infty} \leq C h^{3}\left\|D^{3} f\right\|_{\infty}, \text { where } C<\frac{1}{18}
$$

\subsection{Properties of the Gauss quasi-interpolant}

The specific coefficient forms of the Gauss quadratic spline quasi-interpolant $\widetilde{Q} f=\sum_{i \in J} \mu_{i}(f) B_{i}$, for extreme indices, are the following:

$$
\begin{array}{ll}
\mu_{0}(f):=\alpha_{0} \hat{f}_{0}+\beta_{0} \tilde{f}_{1}+\gamma_{0} \hat{f}_{1}+\delta_{0} \tilde{f}_{2}, & \mu_{n+1}(f):=\alpha_{0} \tilde{f}_{n+1}+\beta_{0} \hat{f}_{n}+\gamma_{0} \tilde{f}_{n}+\delta_{0} \hat{f}_{n-1}, \\
\mu_{1}(f):=\alpha_{1} \hat{f}_{0}+\beta_{1} \tilde{f}_{1}+\gamma_{1} \hat{f}_{1}+\delta_{1} \tilde{f}_{2}, & \mu_{n}(f):=\alpha_{1} \tilde{f}_{n+1}+\beta_{1} \hat{f}_{n}+\gamma_{1} \tilde{f}_{n}+\delta_{1} \hat{f}_{n-1},
\end{array}
$$

where the parameters have the following values $(r=\sqrt{3})$ :

$$
\begin{gathered}
\alpha_{0}=(43-r) / 13, \beta_{0}=-(1+r / 2), \gamma_{0}=-(3-r) / 2, \delta_{0}=(5+2 r) / 26, \\
\alpha_{1}=-(17-r) / 13, \beta_{1}=-\beta_{0}, \gamma_{1}=-\gamma_{0}, \delta_{1}=-\delta_{0}
\end{gathered}
$$

Like $\bar{Q}$, the Gauss QI $\widetilde{Q}$ can be written in the quasi-Lagrange form

$$
\widetilde{Q} f=\sum_{j=1}^{n+1} \tilde{f}_{j} \tilde{B}_{j}+\sum_{j=0}^{n} \hat{f}_{j} \hat{B}_{j}, \text { with }\left\{\begin{array}{l}
\tilde{B}_{j}:=\beta B_{j-1}+\alpha B_{j} \\
\hat{B}_{j}:=\alpha B_{j}+\beta B_{j+1}
\end{array}, 3 \leq j \leq n-2 .\right.
$$

The coefficients in the basis $\left\{B_{i}, i \in J\right\}$ of the first and last five functions are given in the following table (with upper (resp. lower) indices for left (resp. right) functions). 


\begin{tabular}{|c|c|c|c|c|c|}
\hline$i$ & 0 & 1 & 2 & 3 & \\
\hline$\hat{B}_{0}$ & $\alpha_{0}$ & $\alpha_{1}$ & 0 & 0 & $\tilde{B}_{n+1}$ \\
$\tilde{B}_{1}$ & $\beta_{0}$ & $\beta_{1}$ & 0 & 0 & $\hat{B}_{n}$ \\
$\hat{B}_{1}$ & $\gamma_{0}$ & $\gamma_{1}$ & $\beta$ & 0 & $\tilde{B}_{n}$ \\
$\tilde{B}_{2}$ & $\delta_{0}$ & $\delta_{1}$ & $\alpha$ & 0 & $\hat{B}_{n-1}$ \\
$\hat{B}_{2}$ & 0 & 0 & $\alpha$ & $\beta$ & $\tilde{B}_{n-1}$ \\
\hline & $n+1$ & $n$ & $n-1$ & $n-2$ & $i$ \\
\hline
\end{tabular}

As for $\bar{Q}$, it is easy to verify that $\widetilde{Q}$ interpolates $f$ at the points $a$ and $b$, is exact on $\mathbb{P}_{2}$, and has a small infinite norm.

Proposition 4. The quasi-interpolant $\widetilde{Q}$ is exact on the space $\mathbb{P}_{2}$ of quadratic polynomials. Moreover its infinite norm satisfies $\|\widetilde{Q}\|_{\infty} \approx 2.7$

Proof: The infinite norm of the linear operator $\widetilde{Q}$ is equal to the Chebyshev norm of its Lebesgue function $\tilde{\Lambda}:=\sum_{j=1}^{n+1}\left|\tilde{B}_{j}\right|+\sum_{j=0}^{n}\left|\hat{B}_{j}\right|$ and a direct study of the latter leads to $\|\widetilde{Q}\|_{\infty}=|\tilde{\Lambda}|_{\infty} \approx 2.7$.

Proposition 5. For all indices $i$, there holds $\widetilde{Q} f\left(x_{i}\right)-f\left(x_{i}\right)=O\left(h^{4}\right)$ and $\widetilde{Q} f\left(t_{i}\right)-f\left(t_{i}\right)=O\left(h^{4}\right)$, therefore $\widetilde{Q} f$ is superconvergent on both sets of points $\mathcal{X}_{n}$ and $\mathcal{T}_{n}$.

Proof: As in Proposition 2, the superconvergence of $\widetilde{Q} f$ on $\mathcal{X}_{n}$ and $\mathcal{T}_{n}$ is verified by computing Taylor expansions at those points with the help of a computer algebra system. Setting $\tilde{E} f:=\widetilde{Q} f-f$, we thus obtain successively

$$
\begin{aligned}
& \tilde{E} f\left(t_{i}\right) \sim \tilde{C}_{i} h^{4} D^{4} f\left(t_{i}\right), \quad \tilde{E} f\left(x_{i}\right) \sim \tilde{C}_{i}^{\prime} h^{4} D^{4} f\left(x_{i}\right), \text { where } \\
& \tilde{C}_{1}=\tilde{C}_{n}=\frac{61-9 r}{6912}, \tilde{C}_{2}=\tilde{C}_{n-1}=\frac{139-29 r}{6912}, \tilde{C}_{i}=\frac{73-16 r}{1056}, 3 \leq i \leq n-2, \\
& \tilde{C}_{1}^{\prime}=\tilde{C}_{n-1}^{\prime}=\frac{43-5 r}{1728}, \tilde{C}_{i}^{\prime}=\frac{25-4 r}{864}, 2 \leq i \leq n-2 .
\end{aligned}
$$

The values of constants lie between $6 \times 10^{-3}$ and $4 \times 10^{-2}$.

We could also consider the global approximation error $\widetilde{Q} f-f$, which is also $O\left(h^{3}\right)$ for functions $f$ having a bounded third derivative. We do not give details here, the results being quite similar to those of Proposition 3. 


\section{Approximation of first derivatives}

3.1 Superconvergence on the set $\mathcal{G}_{n}$

Proposition 6. For $f \in C^{4}(I)$, the following error estimates hold at Gauss points $\mathcal{G}_{n}$ for both quasi-interpolants $Q=\bar{Q}$ or $\widetilde{Q}$

$$
D Q f\left(u_{i}\right)-D f\left(u_{i}\right)=O\left(h^{3}\right), \quad D Q f\left(v_{i}\right)-D f\left(v_{i}\right)=O\left(h^{3}\right), \quad 1 \leq i \leq n .
$$

As the global approximation order of $D f$ by DQf on the interval is only $O\left(h^{2}\right)$, there appears a superconvergence phenomenon of the first derivatives on $\mathcal{G}_{n}$, which does not hold on $\mathcal{X}_{n}$ and $\mathcal{T}_{n}$.

Proof: As in Propositions 2 and 4 above, the results are verified by computing Taylor expansions on $\mathcal{G}_{n}, \mathcal{X}_{n}$ and $\mathcal{T}_{n}$, with the help of a computer algebra system. We set $\bar{E} f:=\bar{Q} f-f$ and $\tilde{E} f:=\widetilde{Q} f-f$, and we obtain successively

$$
\begin{aligned}
& D \bar{E} f\left(u_{i}\right) \sim \bar{C}_{i} h^{3} D^{4} f\left(u_{i}\right), \quad D \bar{E} f\left(v_{i}\right) \sim \bar{C}_{i}^{\prime} h^{3} D^{4} f\left(v_{i}\right), \quad \text { where } \\
& \bar{C}_{1}=\bar{C}_{n}=\frac{(27-16 r)}{1728}, \bar{C}_{2}=\bar{C}_{n-1}=\frac{(27-23 r)}{1728}, \bar{C}_{i}=-\frac{r}{108}, \quad 3 \leq i \leq n-2, \\
& \bar{C}_{1}^{\prime}=\bar{C}_{n}^{\prime}=\frac{(27+16 r)}{1728}, \bar{C}_{2}^{\prime}=\bar{C}_{n-1}^{\prime}=\frac{(27+23 r)}{1728}, \bar{C}_{i}^{\prime}=-\frac{r}{108}, 3 \leq i \leq n-2, \\
& D \tilde{E} f\left(u_{i}\right) \sim \tilde{C}_{i} h^{3} D^{4} f\left(u_{i}\right), \quad D \tilde{E} f\left(v_{i}\right) \sim \tilde{C}_{i}^{\prime} h^{3} D^{4} f\left(v_{i}\right), \quad \text { where } \\
& \tilde{C}_{1}=\tilde{C}_{n}=\frac{(66-17 r)}{2592}, \tilde{C}_{2}=\tilde{C}_{n-1}=\frac{(6-25 r)}{2592}, \tilde{C}_{i}=-\frac{r}{108}, 3 \leq i \leq n-2, \\
& \tilde{C}_{1}^{\prime}=\tilde{C}_{n}^{\prime}=\frac{(63+2 r)}{2592}, \tilde{C}_{2}^{\prime}=\tilde{C}_{n-1}^{\prime}=\frac{(15+16 r)}{2592}, \tilde{C}_{i}^{\prime}=-\frac{r}{108}, 3 \leq i \leq n-2 .
\end{aligned}
$$

(the values of these constants lie between $4.10^{-4}$ and $4.10^{-2}$ ). Direct computations also show that $D \bar{E} f\left(t_{i}\right), D \bar{E} f\left(x_{i}\right), D \tilde{E} f\left(t_{i}\right), D \tilde{E} f\left(x_{i}\right)$ are only $O\left(h^{2}\right)$

\subsection{Improved global approximation of the first derivative}

While $D Q f$ is a continuous piecewise linear function, a better and smoother global approximant of the first derivative $D f$ is provided by the Gauss quasiinterpolant $\widetilde{Q}(D Q f)$ of $D Q f$, which is a $C^{1}$ quadratic spline. Let us briefly study the global approximation order of $D f$ by $\widetilde{Q}(D Q f)$. We first write

$$
D f-\widetilde{Q}(D Q f)=(D f-\widetilde{Q} D f)+\widetilde{Q}(D f-D Q f) .
$$


From the results of Section 2, the first error satisfies $D f-\widetilde{Q} D f=O\left(h^{3}\right)$ when $f$ has a fourth order bounded derivative. The second error can be written

$$
\widetilde{Q}(D f-D Q f)=\sum_{j \in J}\left(\mu_{j}(D f)-\mu_{j}(D Q f)\right) B_{j} .
$$

The coefficient $\mu_{j}(D f)-\mu_{j}(D Q f)$ being, for all $j \in J$, a linear combination of values of $D f-D Q f$ on $\mathcal{G}_{n}$, we deduce from Proposition 6 that it is a $O\left(h^{3}\right)$. Therefore the same result holds for $\widetilde{Q}(D f-D Q f)$ because of the property $\sum_{j \in J} B_{j}=1$. In fact, there are two close approximants: one is $\widetilde{Q}(D \bar{Q} f)$ (i.e. the Gauss QI of the derivative of the uniform QI) and the other is $\widetilde{Q}(D \widetilde{Q} f)$ (i.e. the Gauss QI of the derivative of the Gauss QI of the given function $f$ ). Numerical experiments show that both approximants are quite close to each other and give a global approximation of $D f$ in $O\left(h^{3}\right)$.

\section{Derivation matrices}

\subsection{Direct derivation}

Let $S=\sum_{j \in J} c_{j} B_{j}$ be a quadratic spline obtained as a quasi-interpolant $Q f$ of a given function $f$. Denoting $\left\{N_{j},-1 \leq j \leq n+1\right\}$ the hat functions with supports $\left[x_{j-1}, x_{j+1}\right]$, we have $S^{\prime}=\sum_{j=0}^{n} \frac{1}{h}\left(c_{j+1}-c_{j}\right) N_{j}$, which implies that

$$
\begin{aligned}
& S^{\prime}(a)=\frac{1}{h}\left(c_{1}-c_{0}\right), S^{\prime}(b)=\frac{1}{h}\left(c_{n+1}-c_{n}\right), \text { and for } 1 \leq i \leq n, \\
& S^{\prime}\left(u_{i}\right)=\frac{1}{h}\left(-\frac{3+r}{6} c_{i-1}+\frac{r}{3} c_{i}+\frac{3-r}{6} c_{i+1}\right), \\
& S^{\prime}\left(v_{i}\right)=\frac{1}{h}\left(-\frac{3-r}{6} c_{i-1}+\frac{r}{3} c_{i}+\frac{3+r}{6} c_{i+1}\right) \\
& S^{\prime}\left(t_{i}\right)=\frac{1}{2}\left(S^{\prime}\left(u_{i}\right)+S^{\prime}\left(v_{i}\right)\right)=\frac{1}{2 h}\left(c_{i+1}-c_{i-1}\right) .
\end{aligned}
$$

When $S=\bar{Q} f$, let $\mathbf{f} \in \mathbb{R}^{n+2}$ be the vector with components $\left\{f_{i}, i \in J\right\}$ and let $\mathbf{f}^{\prime} \in \mathbb{R}^{n+2}$ be the vector with components $\left\{f_{i}^{\prime}=S^{\prime}\left(t_{i}\right), i \in J\right\}$. These two vectors satisfy the equation $\mathbf{f}^{\prime}=\overline{\mathcal{D}} \mathbf{f}$ where the derivation matrix $\overline{\mathcal{D}}$ on $\mathcal{T}_{n}$ is a $(n+2) \times(n+2) 5$-diagonal square matrix.

Similarly, when $S=\widetilde{Q} f$, let $\mathbf{g} \in \mathbb{R}^{2 n+2}$ be the vector with components $\left\{g_{0}:=\right.$ $\left.\hat{f}_{0} ; g_{2 i-1}:=\tilde{f}_{i}, g_{2 i}:=\hat{f}_{i}, 1 \leq i \leq n ; g_{2 n+1}:=\hat{f}_{n+1}\right\}$ and let $\mathbf{g}^{\prime} \in \mathbb{R}^{2 n+2}$ be the vectors with components $\left\{g_{0}^{\prime}:=S^{\prime}(a) ; g_{2 i-1}^{\prime}:=S^{\prime}\left(u_{i}\right), g_{2 i}^{\prime}:=S^{\prime}\left(v_{i}\right), 1 \leq i \leq\right.$ $\left.n ; g_{2 n+1}^{\prime}:=S^{\prime}(b)\right\}$. These two vectors satisfy the equation $\mathbf{g}^{\prime}=\tilde{\mathcal{D}} \mathbf{g}$ where the derivation matrix $\tilde{\mathcal{D}}$ on $\mathcal{G}_{n}$ is a $(2 n+2) \times(2 n+2)$ band matrix. 
However, the approximation order given by direct derivation on $\mathcal{T}_{n}$ is only $O\left(h^{2}\right)$. We will improve on this result in the next subsection.

Example: The following tables give the maximum error on derivatives on $\mathcal{T}_{n}: e_{k}^{\prime}=\max _{i \in J}\left|f^{\prime}\left(t_{i}\right)-f_{i}^{\prime}\right|$, for $f:=\phi_{k}, k=1,2$, computed for various partitions in $n$ subintervals of $I=[0,1]$, with $\phi_{1}(x)=\left(1-x^{2}\right)^{2} / 4$ and $\phi_{2}(x)=$ $0.9 /(\cosh (10 x-2))^{2}+0.8 / \cosh (100 x-60)$. We use the notation $1.1(-2)$ for $1.1 \times 10^{-2}$.

\begin{tabular}{|c|c|c|c|c|}
\hline$n$ & 8 & 16 & 32 & 64 \\
\hline$e_{1}^{\prime}$ & $1.1(-2)$ & $2.8(-3)$ & $7.2(-4)$ & $1.8(-4)$ \\
\hline$n$ & 256 & 512 & 1024 & 2048 \\
\hline$e_{2}^{\prime}$ & 1.2 & $2.2(-1)$ & $5.0(-2)$ & $1.2(-2)$ \\
\hline
\end{tabular}

\subsection{Improved derivation order on $\mathcal{T}_{n}$}

The practical problem appearing in the construction of an efficient derivation matrix is that the superconvergence of derivatives holds on $\mathcal{G}_{n}$ while in general data are given on $\mathcal{T}_{n}$. In this section, we shall improve the approximation error on derivatives up to $O\left(h^{3}\right)$ on $\mathcal{T}_{n}$ by doing convenient linear combinations of values of $D \bar{Q} f\left(t_{i}\right)$. Using again the notations $S:=\bar{Q} f$ and $f_{i}^{\prime}:=S^{\prime}\left(t_{i}\right), i \in J$, we consider the following approximations of $f^{\prime}$ on $\mathcal{T}_{n}$.

$$
\begin{array}{rlrl}
y_{0}^{\prime} & :=\frac{1}{6}\left(8 f_{0}^{\prime}-3 f_{1}^{\prime}+f_{2}^{\prime}\right), & y_{1}^{\prime}:=\frac{1}{12}\left(-2 f_{0}^{\prime}+15 f_{1}^{\prime}-f_{2}^{\prime}\right) \\
y_{j}^{\prime}:=\frac{1}{24}\left(-f_{j-1}^{\prime}+26 f_{j}^{\prime}-f_{j+1}^{\prime}\right), & 2 \leq j \leq n-1 \\
y_{n}^{\prime}:=\frac{1}{12}\left(-f_{n-1}^{\prime}+15 f_{n}^{\prime}-2 f_{n+1}^{\prime}\right), & y_{n+1}^{\prime}:=\frac{1}{6}\left(f_{n-1}^{\prime}-3 f_{n}^{\prime}+8 f_{n+1}^{\prime}\right)
\end{array}
$$

Let us give a short proof of the fact that $f^{\prime}\left(t_{j}\right)-y_{j}^{\prime}$ is at least $O\left(h^{3}\right)$ for $4 \leq j \leq n-3$. Similar, but more complex calculations give the same results for the four first and last indices.

$$
\begin{aligned}
& f_{j-1}^{\prime}=S^{\prime}\left(t_{j-1}\right)=\frac{1}{2 h}\left(\lambda_{j}(f)-\lambda_{j-2}(f)\right)=\frac{1}{16 h}\left(f_{j-3}-10 f_{j-2}+10 f_{j}-f_{j+1}\right) . \\
& f_{j}^{\prime}=S^{\prime}\left(t_{j}\right)=\frac{1}{2 h}\left(\lambda_{j+1}(f)-\lambda_{j-1}(f)\right)=\frac{1}{16 h}\left(f_{j-2}-10 f_{j-1}+10 f_{j+1}-f_{j+2}\right) . \\
& f_{j+1}^{\prime}=S^{\prime}\left(t_{j+1}\right)=\frac{1}{2 h}\left(\lambda_{j+2}(f)-\lambda_{j}(f)\right)=\frac{1}{8 h}\left(f_{j-1}-10 f_{j}+10 f_{j+2}-f_{j+3}\right) .
\end{aligned}
$$


which gives, for $f$ smooth enough, by using Taylor expansions :

$$
f^{\prime}\left(t_{j}\right)-y_{j}^{\prime}:=f^{\prime}\left(t_{j}\right)-\frac{1}{24}\left(-f_{j-1}^{\prime}+26 f_{j}^{\prime}-f_{j+1}^{\prime}\right)=\frac{9 h^{4}}{320} f^{(5)}\left(t_{j}\right)+O\left(h^{6}\right) .
$$

Let $\mathbf{y}^{\prime} \in \mathbb{R}^{n+2}$ be the vector with components $\left\{y_{i}^{\prime}, i \in J\right\}$. The vectors $\mathbf{f}$ and $\mathbf{y}^{\prime}$ satisfy $\mathbf{y}^{\prime}=\hat{\mathcal{D}} \mathbf{f}$. The improved derivation matrix $\hat{\mathcal{D}}$ has the following structure

$$
\left[\begin{array}{cccccccccc}
a_{1} & a_{2} & a_{3} & a_{4} & a_{5} & 0 & 0 & \ldots & & \\
b_{1} & b_{2} & b_{3} & b_{4} & b_{5} & 0 & 0 & 0 & \ldots & \\
c_{1} & c_{2} & c_{3} & c_{4} & -\frac{3}{32} & \frac{1}{384} & 0 & 0 & \ldots & \\
d_{1} & d_{2} & d_{3} & d_{4} & \frac{87}{128} & -\frac{3}{32} & \frac{1}{384} & 0 & \ldots & \\
0 & -\frac{1}{384} & \frac{3}{32} & -\frac{87}{128} & 0 & \frac{87}{128} & -\frac{3}{32} & \frac{1}{384} & 0 & \ldots \\
\ldots & \ldots & \ldots & \ldots & \ldots & \ldots & \ldots & \ldots & \ldots & \ldots \\
\ldots & 0 & -\frac{1}{384} & \frac{3}{32} & -\frac{87}{128} & 0 & \frac{87}{128} & -\frac{3}{32} & \frac{1}{384} & 0 \\
\ldots & 0 & 0 & -\frac{1}{384} & \frac{3}{32} & -\frac{87}{128} & -d_{4} & -d_{3} & -d_{2} & -d_{1} \\
\ldots & 0 & 0 & 0 & -\frac{1}{384} & \frac{3}{32} & -c_{4} & -c_{3} & -c_{2} & -c_{1} \\
\ldots & 0 & 0 & 0 & 0 & -b_{5} & -b_{4} & -b_{3} & -b_{2} & -b_{1} \\
\ldots & 0 & 0 & 0 & 0 & -a_{5} & -a_{4} & -a_{3} & -a_{2} & -a_{1}
\end{array}\right]
$$

where the coefficients at the upper left and lower right corners are given by

$$
\begin{aligned}
& a_{1}=-31 / 10, \quad a_{2}=367 / 96, \quad a_{3}=-29 / 32, \quad a_{4}=31 / 160, \quad a_{5}=-1 / 96, \\
& b_{1}=-21 / 20, \quad b_{2}=89 / 192, \quad b_{3}=45 / 64, \quad b_{4}=-39 / 320, \quad b_{5}=1 / 192 \text {. } \\
& c_{1}=4 / 15, \quad c_{2}=-117 / 128, \quad c_{3}=7 / 96, \quad c_{4}=213 / 320 \text {, } \\
& d_{1}=-1 / 120, \quad d_{2}=13 / 128, \quad d_{3}=-131 / 192, \quad d_{4}=1 / 1920 \text {. }
\end{aligned}
$$

In the examples below, we compare results obtained by using derivatives defined above with those obtained by approximating derivatives by classical finite differences. We use the following finite differences based on $\mathcal{X}_{n}\left(z_{i}=z\left(x_{i}\right)\right)$ :

$$
\begin{aligned}
& z_{0}^{\prime} \approx \frac{1}{h}\left(-\frac{11}{6} z_{0}+3 z_{1}-\frac{3}{2} z_{2}+\frac{1}{3} z_{3}\right), \quad z_{1}^{\prime} \approx \frac{1}{h}\left(-\frac{1}{3} z_{0}-\frac{1}{2} z_{1}+z_{2}-\frac{1}{6} z_{3}\right), \\
& z_{i}^{\prime} \approx \frac{1}{h}\left(\frac{1}{12} z_{i-2}-\frac{2}{3} z_{i-1}+\frac{2}{3} z_{i+1}-\frac{1}{12} z_{i+2}\right), \quad 2 \leq i \leq n-2, \\
& z_{n-1}^{\prime} \approx \frac{1}{h}\left(\frac{1}{3} z_{n}+\frac{1}{2} z_{n-1}-z_{n-2}+\frac{1}{6} z_{n-3}\right), \quad z_{n}^{\prime} \approx \frac{1}{h}\left(\frac{11}{6} z_{n}-3 z_{n-1}+\frac{3}{2} z_{n-2}-\frac{1}{3} z_{n-3}\right) .
\end{aligned}
$$


The errors at the endpoints are in $O\left(h^{3}\right)$ while those at central points are in $O\left(h^{4}\right)$.

Example 2 : The first line (QI) give the maximum errors on the approximate derivatives on $\mathcal{T}_{n}: e_{k}^{\prime}=\max _{i \in J}\left|f^{\prime}\left(t_{i}\right)-y_{i}^{\prime}\right|$, for various values of $n$, and for $f:=\phi_{k}, k=1,2,3$, on $I=[-1,1]$, with $\phi_{1}(x)=\frac{1}{4}\left(1-x^{2}\right)^{2}, \phi_{2}(x):=\frac{1}{1+16 x^{2}}$, $\phi_{3}(x):=\sin (\pi x)+\sin (5 \pi x)$. The second line (FD) gives the estimation of first derivatives given by classical finite differences on $\mathcal{X}_{n}$. Both errors are in $O\left(h^{3}\right)$. The notation $6.5(-3)$ stands for $6.5 \times 10^{-3}$.

\begin{tabular}{|c|c|c|c|c|c|}
\hline$n$ & 8 & 16 & 32 & 64 & 128 \\
\hline$e_{1}^{\prime}(\mathrm{QI})$ & $6.5(-3)$ & $8.1(-4)$ & $1.0(-4)$ & $1.3(-5)$ & $1.6(-6)$ \\
$e_{1}^{\prime}(\mathrm{FD})$ & $2.3(-2)$ & $2.9(-3)$ & $3.7(-4)$ & $4.6(-5)$ & $5.7(-6)$ \\
\hline$e_{2}^{\prime}(\mathrm{QI})$ & 1.3 & 0.32 & $2.8(-2)$ & $2.5(-3)$ & $1.7(-4)$ \\
$e_{2}^{\prime}(\mathrm{FD})$ & 0.12 & 0.22 & $4.0(-2)$ & $3.0(-3)$ & $2.0(-4)$ \\
\hline$e_{3}^{\prime}(\mathrm{QI})$ & 19.4 & 6.7 & 0.71 & $4.9(-2)$ & $3.1(-3)$ \\
$e_{3}^{\prime}(\mathrm{FD})$ & 30.8 & 13.9 & 3.1 & 0.25 & $1.7(-2)$ \\
\hline
\end{tabular}

\subsection{Improved global approximation of the first derivative}

As in section 3.2, a better approximation of the first derivative on $\mathcal{T}_{n}$ provides a better and smoother global approximation of this function on the interval $I$. It suffices to take the quasi-interpolant $\bar{Q}$ on the modified values of derivatives $\left\{y_{j}^{\prime}, j \in J\right\}$ given in the previous section. Let us denote by $\bar{g}^{\prime}$ this QI: $\bar{g}^{\prime}=\sum_{j \in J} y_{j}^{\prime} \bar{B}_{j}$. As the approximation order of $\bar{Q}$ is $O\left(h^{3}\right)$ and $y_{j}^{\prime}-f^{\prime}\left(t_{j}\right)=O\left(h^{3}\right)$, we deduce immediately that, for $f$ smooth enough $\left(D^{4} f\right.$ bounded), $\left\|f^{\prime}-\bar{g}^{\prime}\right\|_{\infty}=O\left(h^{3}\right)$.

Example: The following table give the uniform errors: $e_{k}^{\prime}=\max _{x \in I} \mid f^{\prime}(x)-$ $\bar{g}^{\prime}(x) \mid$, for $f:=\phi_{k}, k=1,2,3$, with the same functions as in previous example, obtained with various partitions in $n$ subintervals of $I=[-1,1]$. The computed errors are at least $O\left(h^{3}\right)$. 


\begin{tabular}{|c|c|c|c|c|c|}
\hline$n$ & 8 & 16 & 32 & 64 & 128 \\
\hline$e_{1}^{\prime}$ & $6.5(-3)$ & $8.1(-4)$ & $1.0(-4)$ & $1.3(-5)$ & $1.6(-6)$ \\
$e_{2}^{\prime}$ & 1.4 & 0.44 & $6.4(-2)$ & $4.9(-3)$ & $3.9(-4)$ \\
$e_{3}^{\prime}$ & 20 & 7.0 & 0.71 & $5.9(-2)$ & $4.0(-3)$ \\
\hline
\end{tabular}

\section{Collocation methods for univariate problems}

Let us consider the univariate Dirichlet problem on $I=[a, b]$ with a differential operator of Sturm-Liouville type :

$$
-D(p(x) D u(x))+r(x) u(x)=f(x), \quad u(a)=u(b)=0,
$$

$p$ being a $C^{1}$ function, $r$ a continuous function and $f$ a piecewise continuous function on $I$. Let $\mathbf{u} \in \mathbb{R}^{n}$ be the vector of unknown values of $u$ on $\mathcal{T}_{n}^{*}:=$ $\mathcal{T}_{n} \backslash\{a, b\}$, and let $\tilde{\mathbf{u}} \in \mathbb{R}^{n+2}$ be the augmented vector with 0 as first and last components. Applying the derivation matrix $\hat{\mathcal{D}}$ gives an approximation $\hat{\mathcal{D}} \tilde{\mathbf{u}} \in \mathbb{R}^{n+2}$ of first derivatives of $u$ on $\mathcal{T}_{n}$. Then, we multiply this vector by the diagonal matrix $P \in \mathbb{R}^{n+2}$ defined by $P(i, i)=p\left(t_{i}\right)$ and we multiply again by the derivation matrix. Denoting by $R \in \mathbb{R}^{n+2}$ the diagonal matrix defined by $R(i, i)=r\left(t_{i}\right)$, we see that the left-hand side of the differential equation is approximated by the vector

$$
\hat{A} \tilde{\mathbf{u}}:=-\hat{\mathcal{D}} P \hat{\mathcal{D}} \tilde{\mathbf{u}}+R \tilde{\mathbf{u}}
$$

Let $A \in \mathbb{R}^{n}$ be the matrix of order $n$ deduced from $\hat{A}$ by deleting the first and last lines and columns, it is well known ([11], chapter 7 ) that the problem is equivalent to solving the linear system $A \mathbf{u}=\mathbf{f}$ where $\mathbf{f} \in \mathbb{R}^{n}$ is the vector of values of $f$ on $\mathcal{T}_{n}^{*}$. Numerical experiments show that the approximation order of the solution is $O\left(h^{3}\right)$.

Example 4. Dirichlet problems $-u^{\prime \prime}=f$ with $u(-1)=u(1)=0$ and successive right hand sides $f=\phi_{k}, k=1,2,3$, with $\phi_{1}(x)=-2+12 x^{2}$, $\phi_{2}(x)=\pi^{2}(\sin (\pi x)+25 \sin (5 \pi x)), \phi_{3}(x)=16 \exp (4 x)$, and corresponding solutions $u=v_{k}: v_{1}(x)=x^{2}\left(1-x^{2}\right), v_{2}(x)=\sin (\pi x)+\sin (5 \pi x), v_{3}(x)=$ $\sinh (4) x+\cosh (4)-\exp (4 x)$. The first line (QI) give the maximum errors $e_{k}:=\max _{i \in J}\left|u\left(t_{i}\right)-\mathbf{u}_{i}\right|, k=1,2,3$, for various values of $n$. The second line (FD) gives the errors obtained by using the derivation matrix associated with 
classical finite differences on $\mathcal{X}_{n}$. Both errors are in $O\left(h^{3}\right)$.

\begin{tabular}{|c|c|c|c|c|c|}
\hline$n$ & 8 & 16 & 32 & 64 & 128 \\
\hline$e_{1}(\mathrm{QI})$ & $4.7(-3)$ & $3.1(-4)$ & $2.0(-5)$ & $1.3(-6)$ & $8.1(-8)$ \\
$e_{1}(\mathrm{FD})$ & $5.0 \mathrm{E}-3$ & $3.1(-4)$ & $2.0(-5)$ & $1.2(-6)$ & $7.7(-8)$ \\
\hline$e_{2}(\mathrm{QI})$ & 17 & 1.9 & $7.5(-2)$ & $3.8(-3)$ & $2.3(-4)$ \\
$e_{2}(\mathrm{FD})$ & 11.4 & 1.9 & $1.3(-1)$ & $7.4(-3)$ & $3.7(-4)$ \\
\hline$e_{3}(\mathrm{QI})$ & 0.84 & 0.10 & $8.7(-3)$ & $6.4(-4)$ & $4.3(-5)$ \\
$e_{3}(\mathrm{FD})$ & 1.11 & 0.11 & $9.0(-3)$ & $7.7(-4)$ & $5.6(-5)$ \\
\hline
\end{tabular}

Example 5. Dirichlet problems $-u^{\prime \prime}+u=f$ with $u(-1)=u(1)=0$ and successive right-hand sides $f=\phi_{k}, k=1,2$, with $\phi_{1}(x)=\left(1+\pi^{2}\right) \sin (\pi x)$, $\phi_{2}(x)=\exp (x / 2)\left(\left(\left(100 \pi^{2}+\frac{3}{4}\right) \sin (10 \pi x)-10 \pi \cos (10 \pi x)\right)+\left(9 \pi^{2}+1\right) \sin (3 \pi x)\right.$, and corresponding solutions $u=v_{k}: v_{1}(x)=\sin (\pi x), v_{2}(x)=\exp (x / 2) \sin (10 \pi x)+$ $\sin (3 \pi x)$. The first line (QI) give the maximum errors $e_{k}:=\max _{i \in J}\left|u\left(t_{i}\right)-\mathbf{u}_{i}\right|$, $k=1,2, \ldots$, for various values of $n$. The second line (FD) gives the errors obtained by using the derivation matrix associated with classical finite differences on $\mathcal{X}_{n}$. Both errors are in $O\left(h^{3}\right)$.

\begin{tabular}{|c|c|c|c|c|c|}
\hline$n$ & 8 & 16 & 32 & 64 & 128 \\
\hline$e_{1}(\mathrm{QI})$ & $1.8(-2)$ & $1.2(-3)$ & $7.6(-5)$ & $4.8(-6)$ & $3.0(-7)$ \\
$e_{1}(\mathrm{FD})$ & $2.3(-2)$ & $1.2(-3)$ & $8.2(-5)$ & $5.3(-6)$ & $3.4(-7)$ \\
\hline$e_{2}(\mathrm{QI})$ & 39 & 22 & 2.9 & 0.12 & $6.3(-3)$ \\
$e_{2}(\mathrm{FD})$ & 41 & 22 & 3.4 & 0.24 & $1.3(-2)$ \\
\hline
\end{tabular}

On these five examples, the solutions obtained by QI are quite comparable with those obtained by FD, and often slightly better. Both they need the solution of a system of linear equations.

\section{Bivariate spline quasi-interpolants on a rectangle}

We briefly sketch the methods developed for bivariate extensions of the previous QIs to functions defined on a domain $\Omega:=[a, b] \times[c, d]$, with uniform partitions $\mathcal{X}_{n}=\left\{x_{j}=a+j h, 0 \leq j \leq m\right\}, \mathcal{Y}_{n}=\left\{y_{k}=c+k h, 0 \leq k \leq n\right\}$, and midpoints $s_{j}=\frac{1}{2}\left(x_{j-1}+x_{j}\right)$ and $t_{k}=\frac{1}{2}\left(y_{k-1}+y_{k}\right)$. We put $J=\{0, \ldots, m+1\}$ and $K=\{0, \ldots, n+1\}$ and define $s_{0}=a, s_{n+1}=b, t_{0}=c, t_{n+1}=d$. We consider tensor products and discrete blending sums [4] of univariate QIs : in 
that case, we get biquadratic QIs. We show that they are superconvergent at all points $\left(\xi_{j}, \eta_{k}\right)$ where $\xi_{j}=x_{j}$ or $s_{j}$ and $\eta_{k}=y_{k}$ or $t_{k}$. Finally, we construct derivation matrices with an application to the solution of the classical bivariate Dirichlet problem $-\Delta u=f$ by associated collocation methods. In this section, $\mathbb{P}_{n}$ denotes the space of polynomials of total degree at most $n$ and $\mathbb{P}_{p, q}$ the space of polynomials of partial degrees at most $p$ in $x$ and $q$ in $y$. Monomials are denoted $m_{p, q}(x, y):=x^{p} y^{q}=m_{p}(x) m_{q}(y)$.

\subsection{Tensor product and discrete blending sum of univariate QIs}

The tensor product $T=P_{2} Q_{2}$ of the two univariate quadratic spline QIs :

$$
P_{2} f(x):=\sum_{j \in J} f\left(s_{j}\right) \bar{B}_{j}(x), \quad Q_{2} g(y):=\sum_{k \in K} g\left(t_{k}\right) \bar{B}_{k}(y),
$$

is the bivariate QI defined by

$$
T F(x, y):=\sum_{(j, k) \in J \times K} F\left(s_{j}, t_{k}\right) \bar{B}_{j, k}(x, y), \text { with } \bar{B}_{j, k}(x, y):=\bar{B}_{j}(x) \bar{B}_{k}(y) .
$$

Proposition 7. The quasi-interpolant $T$ is exact on the space $\mathbb{P}_{2,2}$ of biquadratic polynomials. Moreover $\|T\|_{\infty}=9 / 4$.

Proof. The proof is classical and based on similar techniques to those used e.g. in [2] or [4].

The discrete blending sum (see $[2,4]$ ) of the four quadratic spline QIs :

$$
\begin{aligned}
P_{1} f(x) & :=\sum_{j \in J} f\left(s_{j}\right) B_{j}(x), \quad P_{2} f(x):=\sum_{j \in J} f\left(s_{j}\right) \bar{B}_{j}(x), \\
Q_{1} g(y) & :=\sum_{k \in K} g\left(t_{k}\right) B_{k}(y), \quad Q_{2} g(y):=\sum_{k \in K} g\left(t_{k}\right) \bar{B}_{k}(y),
\end{aligned}
$$

is the bivariate QI defined by

$$
R F(x, y):=\left(P_{1} Q_{2}+P_{2} Q_{1}-P_{1} Q_{1}\right) F(x, y)=\sum_{(j, k) \in J \times K} F\left(s_{j}, t_{k}\right) \tilde{B}_{j, k}(x, y)
$$

where the basic functions $\tilde{B}_{j, k}$ are given by

$$
\tilde{B}_{j, k}(x, y):=B_{j}(x) \bar{B}_{k}(y)+\bar{B}_{j}(x) B_{k}(y)-B_{j}(x) B_{k}(y) .
$$


As $P_{1}$ and $Q_{1}$ are exact on $\mathbb{P}_{1}$, and $P_{2}$ and $Q_{2}$ are exact on $\mathbb{P}_{2}$, it is easy to verify that $R$ is exact on $\mathbb{P}_{1,2} \oplus \mathbb{P}_{2,1}$ which contains $\mathbb{P}_{2}$. Indeed, for all pairs $(p, q)$ with $0 \leq p, q \leq 2$ and $(p, q) \neq(2,2)$, we have:

$$
R m_{p, q}=\left(P_{1} m_{p}\right) m_{q}+m_{p}\left(Q_{1} m_{q}\right)-\left(P_{1} m_{p}\right)\left(Q_{1} m_{q}\right)=m_{p, q} .
$$

For $p \leq 1, P_{1} m_{p}=m_{p}$, therefore $R m_{p, q}=m_{p} m_{q}+m_{p}\left(Q_{1} m_{q}\right)-m_{p}\left(Q_{1} m_{q}\right)=$ $m_{p, q}$. The proof is similar for $q \leq 1$. In addition, from $\left\|P_{2}\right\|_{\infty}=\left\|Q_{2}\right\|_{\infty}=\frac{3}{2}$ and $\left\|P_{1}\right\|_{\infty}=\left\|Q_{1}\right\|_{\infty}=1$, we deduce that $\|R\|_{\infty} \leq 4$. So, we have proved the following:

Proposition 8. The quasi-interpolant $R$ is exact on the space of polynomials $\mathbb{P}_{1,2} \oplus \mathbb{P}_{2,1}$. Moreover $\|R\|_{\infty} \leq 4$.

\subsection{Superconvergence properties}

Superconvergence means exact interpolation for monomials of partial degree at most 3, i.e. $\left(P_{2} m_{p}-m_{p}\right)\left(\xi_{j}\right)=0$ and $\left(Q_{2} m_{q}-m_{q}\right)\left(\eta_{k}\right)=0$ for $0 \leq p, q \leq 3$, $\xi_{j}=x_{j}$ or $s_{j}, \eta_{k}=y_{k}$ or $t_{k}$. Therefore

$$
\left(T m_{p, q}-m_{p, q}\right)\left(\xi_{j}, \eta_{k}\right)=P_{2} m_{p}\left(\xi_{j}\right) Q_{2} m_{q}\left(\eta_{k}\right)-m_{p}\left(\xi_{j}\right) m_{q}\left(\eta_{k}\right)=0,
$$

i.e. $T$ is superconvergent at all gridpoints $\left(\xi_{j}, \eta_{k}\right)$. For the operator $R$, we get

$$
\begin{aligned}
\left(R m_{p, q}\right. & \left.-m_{p, q}\right)\left(\xi_{j}, \eta_{k}\right)=P_{1} m_{p}\left(\xi_{j}\right) m_{q}\left(\eta_{k}\right)+m_{p}\left(\xi_{j}\right) Q_{1} m_{q}\left(\eta_{k}\right) \\
& -P_{1} m_{p}\left(\xi_{j}\right) Q_{1} m_{q}\left(\eta_{k}\right)-m_{p}\left(\xi_{j}\right) m_{q}\left(\eta_{k}\right) \\
& =\left(P_{1} m_{p}\left(\xi_{j}\right)-m_{p}\left(\xi_{j}\right)\right)\left(m_{q}\left(\eta_{k}\right)-Q_{1} m_{q}\left(\eta_{k}\right)\right)=O\left(h^{4}\right),
\end{aligned}
$$

since $P_{1} f-f=O\left(h^{2}\right)$ and $Q_{1} g-g=O\left(h^{2}\right)$ for smooth functions $f$ and $g$. Thus, there is no exact interpolation, but the approximation order is four. By Taylor's formula, this also implies that $(R F-F)\left(\xi_{j}, \eta_{k}\right)=O\left(h^{4}\right)$ for all smooth bivariate functions $F$. The superconvergence of derivatives will be studied elsewhere.

\section{Bivariate Dirichlet problem}

In order to illustrate the possibilities of the bivariate collocation method deduced from tensor product quasi-interpolation, we consider the classical model 
problem $-\Delta u=f$ on a square domain $\Omega$ with Dirichlet boundary conditions. As discrete approximation of the Laplacian, we use the Kronecker product of matrices (see [11], chapter 7) $K=A \otimes I+I \otimes A$, where $A$ is the matrix deduced from the derivation matrix $\hat{\mathcal{D}}$ (Sections 4.2 and 5 ). In that case, denoting by $\mathbf{u}$ the vector of components of the solution in the basis of tensor-product Bsplines and by $\mathbf{f}$ the vector of values of $f$ at the interior points of $\Omega$, the linear system to solve is then $-K \mathbf{u}=\mathbf{f}$.

Examples. Let the right-hand sides be $f_{1}=4\left(1-3 x^{2}\right)\left(1-y^{2}\right)^{2}+4(1-$ $\left.3 y^{2}\right)\left(1-x^{2}\right)^{2}$ on $\Omega=[-1,1]^{2}$ and $f_{2}=64\left[((2 x-1) y(y-1))^{2}+((2 y-1) x(x-\right.$ $\left.1))^{2}\right] \sin (4 x(x-1) y(y-1))-32[x(x-1)+y(y-1)] \cos (4 x(x-1) y(y-1))$ on $\Omega=[0,1]^{2}$. The corresponding exact solutions are respectively

$u_{1}=\left(\left(1-x^{2}\right)\left(1-y^{2}\right)\right)^{2}$ and $u_{2}=4 \sin (4 x(x-1) y(y-1))$. The first line (QI) give the maximum errors $e_{1}$ and $e_{2}$ at the points of $\mathcal{T}_{n} \times \mathcal{T}_{n}$ for various values of $n$ (we give between brackets the numerical convergence order). The second line (FD) gives the errors obtained by using the derivation matrix associated with classical finite differences on $\mathcal{X}_{n}$.

\begin{tabular}{|c|c|c|c|c|c|c|}
\hline$n$ & 8 & 16 & 24 & 32 & 40 & 48 \\
\hline$e_{1}(\mathrm{QI})$ & $3.9(-3)$ & $2.8(-4)[3.8]$ & $5.8(-5)[3.9]$ & $1.9(-5)[3.9]$ & $7.9(-6)[3.9]$ & $3.8(-6)[3.9]$ \\
$e_{1}(\mathrm{FD})$ & $7.6(-3)$ & $4.2(-4)[4.2]$ & $7.8(-5)[4.1]$ & $2.4(-5)[4.1]$ & $9.7(-6)[4.1]$ & $4.6(-6)[4.1]$ \\
\hline$e_{2}(\mathrm{QI})$ & $2.3(-4)$ & $2.4(-5)[3.3]$ & $5.4(-6)[3.6]$ & $1.9(-6)[3.6]$ & $8.4(-7)[3.7]$ & $4.2(-7)[3.8]$ \\
$e_{2}(\mathrm{FD})$ & $2.5(-4)$ & $4.9(-5)[2.3]$ & $1.2(-5)[3.4]$ & $4.3(-6)[3.7]$ & $1.8(-6)[3.7]$ & $9.3(-7)[3.8]$ \\
\hline
\end{tabular}

Remark. We get convergence orders which seem closer to 4 than to 3 . This is due to the fact that derivatives are approximated to the 4th order at most points inside the domain (see Section 4).

\section{References}

[1a] C. de Boor, Splines as linear combinations of B-splines, a survey, in: G. G. Lorentz et al. (Eds.), Approximation Theory II, Academic Press, NewYork, 1976, pp 1-47.

[1b] C. de Boor, A practical guide to splines, Springer-Verlag, Berlin, New-York, 2001 (revised edition).

[1c] C. de Boor, Quasi interpolants and approximation power of multivariate splines, in: W. Dahmen et al. (Eds.), Computation of Curves and Surfaces, Kluwer, Dordrecht, 1990, pp. 313-345. 
[2] W. Cheney, W. Light, A course in approximation theory, Brooks/Cole, 2000.

[3] C. K. Chui, Multivariate splines, SIAM, Philadelphia, 1988.

[4] F. J. Delvos, W. Schempp, Boolean methods in interpolation and approximation, Pitman Research Notes in Maths Series 230, Longman Scientific and Technical, Harlow, 1989.

[5] R. A. DeVore, G. G. Lorentz, Constructive Approximation, Springer-Verlag, Berlin, New-York, Berlin,1993.

[6a] B. Fornberg, A practical guide to pseudospectral methods, Cambridge University Press, 1998.

[6b] B. Fornberg, D. M. Sloan, A review of pseudospectral methods for solving partial differential equations, in A. Iserles (Ed.), Acta Numerica 1994, Cambridge University Press, 1994, pp. 203-267.

[7a] F. Foucher, P. Sablonnière, Approximating partial derivatives of first and second order by quadratic spline quasi-interpolants on uniform meshes, Math. Comput. Simul. 77(2008), 202-208.

[7b] F. Foucher, P. Sablonnière, Superconvergence properties of some bivariate $C^{1}$ quadratic spline quasi-interpolants, in: A. Cohen, J.-L. Merrien, L. .L Schumaker (Eds.), Curves and Surfaces Fitting: Avignon 2006, Nashboro Press, Brentwood, 2007, pp. 160-169.

[8] T. Lyche and L. L. Schumaker, Local spline approximation methods, J. Approx. Theory 4 (1975) 294-325.

[9a] P. Sablonnière, Quadratic spline quasi-interpolants on bounded domains of $\mathbb{R}^{d}$, $d=1,2,3$, Rend. Sem. Mat. Univ. Pol. Torino 61 (2003) 229-246.

[9b] P. Sablonnière, Univariate spline quasi-interpolants and applications to numerical analysis, Rend. Sem. Mat. Univ. Pol. Torino 63 (2005) 107-118.

[9c] P. Sablonnière, Recent progress on univariate and multivariate polynomial and spline quasi-interpolants, in: M. G. De Bruijn et al. (Eds.), Trends and applications in constructive approximation, ISNM Vol. 151, BV (2005) 229245.

[10] L. L. Schumaker, Spline functions: basic theory, John Wiley \& Sons, New-York, 1981.

[11] L. N. Trefethen, Spectral methods in Matlab, SIAM, Philadelphia, 2000. 